\title{
ON THE CONNECTEDNESS OF PLANAR SELF-AFFINE SETS
}

\author{
JING-CHENG LIU ${ }^{1}$, JUN JASON LUO ${ }^{2}$ AND HENG-WEN XIE ${ }^{1}$
}

\begin{abstract}
In this paper, we consider the connectedness of planar self-affine set $T(A, \mathcal{D})$ arising from an integral expanding matrix $A$ with characteristic polynomial $f(x)=x^{2}+b x+c$ and a digit set $\mathcal{D}=\{0,1, \ldots, m\} v$. The necessary and sufficient conditions only depending on $b, c, m$ are given for the $T(A, \mathcal{D})$ to be connected. Moreover, we also consider the case that $\mathcal{D}$ is non-consecutively collinear.
\end{abstract}

\section{Introduction}

Let $A \in M_{n}(\mathbb{Z})$ be an expanding $n \times n$ integral matrix, i.e., all eigenvalues of $A$ have moduli strictly greater than 1 . Let $\mathcal{D}=\left\{d_{1}, \ldots, d_{m}\right\}$ be a finite set of $m$ distinct vectors on $\mathbb{R}^{n}$. We call $\mathcal{D}$ a digit set. Then the maps

$$
S_{i}(x)=A^{-1}\left(x+d_{i}\right), \quad 1 \leq i \leq m,
$$

are contractive under a suitable norm in $\mathbb{R}^{n}[15]$, and it is well-known that there exists a unique non-empty compact set $T:=T(A, \mathcal{D})$ satisfying the set-valued functional equation

$$
T=\bigcup_{i=1}^{m} S_{i}(T)
$$

Usually $T$ can also be written as

$$
T=A^{-1}(T+\mathcal{D})=\left\{\sum_{i=1}^{\infty} A^{-1} d_{j_{i}}: d_{j_{i}} \in \mathcal{D}\right\} .
$$

The $T$ is called the self-affine set (or attractor) of the iterated function system (IFS) $\left\{S_{i}\right\}_{i=1}^{m}$. We call $T$ a self-affine tile if it has positive Lebesgue measure and

Date: March 29, 2022.

2010 Mathematics Subject Classification. Primary 28A80; Secondary 52C20, 52C45.

Key words and phrases. Connectedness, digit set, self-affine set, self-affine tile, neighbor.

The research is supported in part by the NNSF of China (No.11171100, No.11301175, No.11301322), the Hunan Provincial NSF (No.13JJ4042), the Special Fund for Excellent Doctoral Dissertation of Hunan Provincial (No.YB2012B025), Specialized Research Fund for the Doctoral Program of Higher Education of China (20134402120007), Foundation for Distinguished Young Talents in Higher Education of Guangdong (2013LYM_0028) and STU Scientific Research Foundation for Talents (NTF12016). 
the union in (1.1) is essentially disjoint, i.e., the intersection $\left(T+d_{i}\right) \cap\left(T+d_{j}\right)$ has zero Lebesgue measure for $i \neq j$. In this situation, $T^{\circ} \neq \emptyset$ and $c:=|\operatorname{det}(A)|=m$.

The study on the topological properties of self-affine sets/tiles $T(A, \mathcal{D})$ on $\mathbb{R}^{n}$ is an interesting topic in fractal geometry, tiling theory and even canonical number systems. In particular, connectedness, disk-likeness (i.e., homeomorphic to a closed disk in the case $n=2$ ) and the boundary structures have been investigated extensively. Gröchenig and Haas [4] as well as Hacon et al. [5] discussed a few special connected self-affine tiles. Lau and his coworkers ([7], [9], [10], [11]) systematically studied a large class of connected self-affine tiles arising from so-called consecutive collinear digit set, i.e., of the form $\mathcal{D}=\{0,1, \ldots, c-1\} v$, and their disk-likeness in the plane. They observed an algebraic property of the characteristic polynomial of $A$ to determine the connectedness of $T(A, \mathcal{D})$. Akiyama and Gjini [1] also focused on this algebraic property by canonical number systems. On the other hand, Bandt and Wang [2] and Leung and one of the authors [14] also concerned the disk-like self-affine tiles or the boundary properties by using a technique of neighbor graphs.

Recently, on $\mathbb{R}^{2}$, Kirat [8] and Deng and Lau [3] found out the connected selfaffine tiles $T(A, \mathcal{D})$ among classes of data $(A, \mathcal{D})$ with non-collinear digit sets $\mathcal{D}$ and characterized the disk-like ones. Leung and one of the authors ([12], [13]) were also interested in the collinear digit set $\{0,1, m\} v$ and the non-collinear digit set $\{0, v, m A v\}$ with the restriction of $|\operatorname{det} A|=3$.

In this paper, we study more general self-affine sets $T(A, \mathcal{D})$ on $\mathbb{R}^{2}$ arising from an integral expanding matrix $A$ with characteristic polynomial $f(x)=x^{2}+b x+c$ and the consecutive collinear digit set $\mathcal{D}=\{0,1, \ldots, m\} v$. We obtain the following main results.

Theorem 1.1. Let the characteristic polynomial of $A$ be $f(x)=x^{2}+b x+c$ and $a$ digit set $\mathcal{D}=\{0,1, \ldots, m\} v$ where $m \geq 1$ and $v \in \mathbb{R}^{2}$ such that $\{v$, Av $\}$ are linearly independent. If $\Delta=b^{2}-4 c \geq 0$ and the eigenvalues of $A$ have moduli $\geq 2$, then

(i) if $c=4$, then $T(A, \mathcal{D})$ is connected if and only if $m \geq 2$;

(ii) otherwise $c \neq 4$, then $T(A, \mathcal{D})$ is connected if and only if

$$
m \geq \begin{cases}\max \{c-|b|+1,|b|-1\} & c>0 \\ |c|-|b|-1 & c<0 .\end{cases}
$$

If $\Delta=b^{2}-4 c<0$, the eigenvalues of $A$ are complex numbers, the self-affine set $T(A, \mathcal{D})$ becomes very complicated. However, under certain situations, we still obtain some interesting results.

Theorem 1.2. Let the characteristic polynomial of $A$ be $f(x)=x^{2}+b x+c$ and $a$ digit set $\mathcal{D}=\{0,1, \ldots, m\} v$ where $m \geq 1$ and $v \in \mathbb{R}^{2}$ such that $\{v$, Av $\}$ are linearly 
independent. If $\Delta=b^{2}-4 c<0$, then $T(A, \mathcal{D})$ is connected if and only if

$$
m \geq \begin{cases}\max \{c-|b|+1,|b|-1\} & b^{2}=3 c \\ c-|b|+1 & b^{2}=2 c, b^{2}=c \\ c-1 & b=0 .\end{cases}
$$

On the other hand, when the characteristic polynomial of $A$ is of the special form $f(x)=x^{2}-(p+q) x+p q$ where $|p|,|q| \geq 2$ are integers, and the digit set $\mathcal{D}$ may be non-consecutively collinear. By letting $f_{1}(x)=x^{2} \pm 4 x+4$ and $f_{2}(x)=$ $x^{2} \pm 7 x+12$, we can characterize the connectedness of the associated self-affine tile $T(A, \mathcal{D})$ through the following theorem, which is also a generalization of [12].

Theorem 1.3. Let the characteristic polynomial of $A$ be $f(x)=x^{2}-(p+q) x+p q$ and $a$ digit set $\mathcal{D}=\{0,1, \ldots,|p q|-2,|p q|-1+s\}$ v where $s \geq 0,|p|,|q| \geq 2$ are integers and $v \in \mathbb{R}^{2}$ such that $\{v, A v\}$ are linearly independent. Then

(i) if $f \neq f_{1}, f_{2}$, then $T(A, \mathcal{D})$ is connected if and only if $s=0$;

(ii) if $f=f_{1}$ or $f_{2}$, then $T(A, \mathcal{D})$ is connected if and only if $s=0$ or 1 .

As in the papers previously cited, a lot of calculations are needed in the proofs. But the main methods are algebraic and make full use of the properties of the matrix $A$. We also provide many figures to illustrate our results.

The paper is organized as follows: In Section 2, we recall several well-known results on the connectedness of self-affine sets and prove a basic lemma; Theorems 1.1 and 1.2 are proved in Section 3, and conclude with an open problem; Theorem 1.3 is proved in Section 4.

\section{Preliminaries}

In the section, we provide several elementary results on self-affine sets $T(A, \mathcal{D})$. We call the digit set $\mathcal{D}$ collinear if $\mathcal{D}=\left\{d_{1}, \ldots, d_{m}\right\} v$ for some non-zero vector $v \in \mathbb{R}^{n}$ and $d_{1}<d_{2}<\cdots<d_{m}, d_{i} \in \mathbb{R}$; If $d_{i+1}-d_{i}=1$, then $\mathcal{D}$ is called a consecutive collinear digit set. Let $D=\left\{d_{1}, \ldots, d_{m}\right\}, \Delta D=D-D=\left\{d=d_{i}-d_{j}: d_{i}, d_{j} \in D\right\}$. Then $\mathcal{D}=D v$ and $\Delta \mathcal{D}=\Delta D v$. It is easy to see that the connectedness of $T(A, \mathcal{D})$ is invariant under a translation of the digit set, hence we always assume that $d_{1}=0$ for simplicity. The following criterion for connectedness of $T(A, \mathcal{D})$ was due to [6] or $[9]$.

Lemma 2.1. A self-affine set $T(A, \mathcal{D})$ with a consecutive collinear digit set $\mathcal{D}=$ $\{0,1, \ldots, m\} v$ is connected if and only if $v \in T-T$. 
Let $\mathbb{Z}[x]$ be the set of polynomials with integer coefficients. A polynomial $f(x) \in$ $\mathbb{Z}[x]$ is said to be expanding if all its roots have moduli strictly bigger than 1 . Note that a matrix $A \in M_{n}(\mathbb{Z})$ is expanding if and only if its characteristic polynomial is expanding. We say that a monic polynomial $f(x) \in \mathbb{Z}[x]$ with $|f(0)|=c$ has the Height Reducing Property (HRP) if there exists $g(x) \in \mathbb{Z}[x]$ such that

$$
g(x) f(x)=x^{k}+a_{k-1} x^{k-1}+\cdots+a_{1} x \pm c
$$

where $\left|a_{i}\right| \leq c-1, i=1, \ldots, c-1$.

This property was introduced by Kirat and Lau [9] to study the connectedness of self-affine tiles with consecutive collinear digit sets. It was proved that:

Proposition 2.2. Let $A \in M_{n}(\mathbb{Z})$ with $|\operatorname{det}(A)|=c$ be expanding and $\mathcal{D}=$ $\{0,1,2, \ldots,(c-1)\} v$. If the characteristic polynomial of $A$ has the Height Reducing Property, then $T(A, \mathcal{D})$ is connected.

In [10], Kirat et al. conjectured that all expanding integer monic polynomials have HRP. Akiyama and Gjini [1] confirmed it up to $n=4$. But it is still unclear for the higher dimensions. Recently, He et al. [7] developed an algorithm of polynomials about HRP. It may be a good attempt on this problem.

Denote the characteristic polynomial of $A$ by $f(x)=x^{2}+b x+c$, where $b, c \in \mathbb{Z}$. We can regard $A$ as the companion matrix of $f(x)$, i.e.,

$$
A=\left[\begin{array}{ll}
0 & -c \\
1 & -b
\end{array}\right]
$$

Let $\Delta=b^{2}-4 c$ be the discriminant. Define $\alpha_{i}, \beta_{i}$ by

$$
A^{-i} v=\alpha_{i} v+\beta_{i} A v, \quad i=1,2, \ldots
$$

According to the Hamilton-Cayley theorem $f(A)=A^{2}+b A+c I=0$ where $I$ is a $2 \times 2$ identity matrix, the following consequence is well-known (please refer to [12], [13]).

Lemma 2.3. Let $\alpha_{i}, \beta_{i}$ be defined as the above. Then $c \alpha_{i+2}+b \alpha_{i+1}+\alpha_{i}=0$ and $c \beta_{i+2}+b \beta_{i+1}+\beta_{i}=0$, i.e.,

$$
\left[\begin{array}{l}
\alpha_{i+1} \\
\alpha_{i+2}
\end{array}\right]=\left[\begin{array}{rr}
0 & 1 \\
-1 / c & -b / c
\end{array}\right]^{i}\left[\begin{array}{l}
\alpha_{1} \\
\alpha_{2}
\end{array}\right] ; \quad\left[\begin{array}{l}
\beta_{i+1} \\
\beta_{i+2}
\end{array}\right]=\left[\begin{array}{rr}
0 & 1 \\
-1 / c & -b / c
\end{array}\right]^{i}\left[\begin{array}{l}
\beta_{1} \\
\beta_{2}
\end{array}\right]
$$

and $\alpha_{1}=-b / c, \alpha_{2}=\left(b^{2}-c\right) / c^{2} ; \beta_{1}=-1 / b, \beta_{2}=b / c^{2}$. Moreover for $\Delta \neq 0$, we have

$$
\alpha_{i}=\frac{c\left(r_{1}^{i+1}-r_{2}^{i+1}\right)}{\Delta^{1 / 2}} \quad \text { and } \quad \beta_{i}=\frac{-\left(r_{1}^{i}-r_{2}^{i}\right)}{\Delta^{1 / 2}}
$$

where $r_{1}=\frac{-b+\Delta^{1 / 2}}{2 c}$ and $r_{2}=\frac{-b-\Delta^{1 / 2}}{2 c}$ are the two roots of $c x^{2}+b x+1=0$. 
Set

$$
\tilde{\alpha}:=\sum_{i=1}^{\infty}\left|\alpha_{i}\right|, \quad \tilde{\beta}:=\sum_{i=1}^{\infty}\left|\beta_{i}\right| .
$$

Then $\tilde{\alpha}$ and $\tilde{\beta}$ are finite numbers as $r_{1}, r_{2}$ have moduli strictly less than 1 .

Write $L:=\{\gamma v+\delta A v: \gamma, \delta \in \mathbb{Z}\}$, then $L$ is a lattice generated by $\{v, A v\}$. For $l \in L \backslash\{0\}$, we call $T+l$ a neighbor of $T$ if $T \cap(T+l) \neq \emptyset$. It is clear that $T+l$ is a neighbor of $T$ if and only if $l \in T-T$, hence $l$ can be expressed as

$$
l=\sum_{i=1}^{\infty} b_{i} A^{-i} v \in T-T, \text { where } b_{i} \in \Delta D .
$$

If $T+l$ is a neighbor of $T$ where $l=\sum_{i=1}^{\infty} b_{i} A^{-i} v:=\gamma v+\delta A v$, then

$$
|\gamma| \leq \max _{i}\left|b_{i}\right| \tilde{\alpha} \quad \text { and } \quad|\delta| \leq \max _{i}\left|b_{i}\right| \tilde{\beta}
$$

By multiplying $A$ on both sides of the expression of $l$ and by using $f(A)=0$, it follows that $T-\left(c \delta+b_{1}\right) v+(\gamma-b \delta) A v$ is also a neighbor of $T$. Repeatedly applying this neighbor-generating algorithm, we then can construct a sequence of neighbors: $\left\{T+l_{n}\right\}_{n=0}^{\infty}$, where $l_{0}=l, l_{n}=\gamma_{n} v+\delta_{n} A v, n \geq 1$ and

$$
\left[\begin{array}{l}
\gamma_{n} \\
\delta_{n}
\end{array}\right]=A^{n}\left[\begin{array}{l}
\gamma \\
\delta
\end{array}\right]-\sum_{i=1}^{n} A^{i-1}\left[\begin{array}{r}
b_{n+1-i} \\
0
\end{array}\right]
$$

Moreover, $\left|\gamma_{n}\right| \leq \max _{i}\left|b_{i}\right| \tilde{\alpha}$ and $\left|\delta_{n}\right| \leq \max _{i}\left|b_{i}\right| \tilde{\beta}$ hold for any $n \geq 0$.

Lemma 2.4. If the characteristic polynomial of the expanding matrix $A$ is $x^{2}+b x+c$ and that of $B$ is $x^{2}-b x+c$. Then the self-affine set $T(A, \mathcal{D})$ is connected if and only if $T(B, \mathcal{D})$ is connected where $\mathcal{D}$ is a consecutive collinear digit set.

Proof. Let $B=-A$ and $T_{1}=T(A, \mathcal{D}), T_{2}=T(-A, \mathcal{D})$. If $l \in T_{1}-T_{1}$, then

$$
l=\sum_{i=1}^{\infty} b_{i} A^{-i} v=\sum_{i=1}^{\infty} b_{2 i}(-A)^{-2 i} v+\sum_{i=1}^{\infty}\left(-b_{2 i-1}\right)(-A)^{-2 i+1} v .
$$

Thus $l \in T_{2}-T_{2}$, and vice versa.

To get the necessary conditions of Theorems 1.1, 1.2 and 1.3 , we need the exact values of $\tilde{\alpha}$ and $\tilde{\beta}$.

Lemma 2.5. Let the characteristic polynomial of the expanding matrix $A$ be $f(x)=$ $x^{2}+b x+c$, where $b, c$ are integers and $\Delta=b^{2}-4 c \geq 0$. Then

$$
\tilde{\alpha}=\left\{\begin{array}{ll}
\frac{|b|-1}{c-|b|+1} & c>0 \\
\frac{|b|+1}{|c|-|b|-1} & c<0 ;
\end{array} \quad \tilde{\beta}= \begin{cases}\frac{1}{c-|b|+1} & c>0 \\
\frac{1}{|c|-|b|-1} & c<0 .\end{cases}\right.
$$


Proof. Let $x_{1}, x_{2}$ denote the roots of $x^{2}+b x+c=0$.

(1) $c>0$. If $\left|x_{1}\right|>\left|x_{2}\right|$, then $\left|\alpha_{i}\right|=\frac{c}{\left|x_{1}-x_{2}\right|}\left(\frac{1}{\left|x_{2}\right|^{i+1}}-\frac{1}{\left|x_{1}\right|^{i+1}}\right),\left|\beta_{i}\right|=$ $\frac{1}{\left|x_{1}-x_{2}\right|}\left(\frac{1}{\left|x_{2}\right|^{i}}-\frac{1}{\left|x_{1}\right|^{i}}\right)$. Hence

$\tilde{\alpha}=\sum_{i=1}^{\infty}\left|\alpha_{i}\right|=\sum_{i=1}^{\infty} \frac{c}{\left|x_{1}-x_{2}\right|}\left(\frac{1}{\left|x_{2}\right|^{i+1}}-\frac{1}{\left|x_{1}\right|^{i+1}}\right)=\frac{\left|x_{1}+x_{2}\right|-1}{\left(\left|x_{1}\right|-1\right)\left(\left|x_{2}\right|-1\right)}=\frac{|b|-1}{c-|b|+1} ;$

$\tilde{\beta}=\sum_{i=1}^{\infty}\left|\beta_{i}\right|=\sum_{i=1}^{\infty} \frac{1}{\left|x_{1}-x_{2}\right|}\left(\frac{1}{\left|x_{2}\right|^{i}}-\frac{1}{\left|x_{1}\right|^{i}}\right)=\frac{1}{\left(\left|x_{1}\right|-1\right)\left(\left|x_{2}\right|-1\right)}=\frac{1}{c-|b|+1}$.

Similarly for $\left|x_{2}\right|>\left|x_{1}\right|$.

If $\left|x_{1}\right|=\left|x_{2}\right|=|b| / 2$, by Lemma 2.3 and a simple calculation, it follows that $\left|\alpha_{i}\right|=\frac{i+1}{\left|x_{1}\right|^{i}}$ and $\left|\beta_{i}\right|=\frac{i}{\left|x_{1}\right|^{i+1}}$. Thus

$$
\begin{aligned}
\left(1-\frac{1}{\left|x_{1}\right|}\right) \tilde{\alpha} & =\sum_{i=1}^{\infty} \frac{i+1}{\left|x_{1}\right|^{i}}-\sum_{j=1}^{\infty} \frac{j+1}{\left|x_{1}\right|^{j+1}}=\frac{2}{\left|x_{1}\right|}+\sum_{\ell=1}^{\infty} \frac{1}{\left|x_{1}\right|^{\ell+1}} \\
& =\frac{2}{\left|x_{1}\right|}+\frac{1}{\left|x_{1}\right|\left(\left|x_{1}\right|-1\right)}=\frac{2\left|x_{1}\right|-1}{\left|x_{1}\right|\left(\left|x_{1}\right|-1\right)} ; \\
\left(\left|x_{1}\right|-1\right) \tilde{\beta} & =\sum_{i=1}^{\infty} \frac{i}{\left|x_{1}\right|^{i}}-\sum_{j=1}^{\infty} \frac{j}{\left|x_{1}\right| j+1}=\frac{1}{\left|x_{1}\right|}+\sum_{\ell=1}^{\infty} \frac{1}{\left|x_{1}\right|^{\ell+1}} \\
& =\frac{1}{\left|x_{1}\right|}+\frac{1}{\left|x_{1}\right|\left(\left|x_{1}\right|-1\right)}=\frac{1}{\left(\left|x_{1}\right|-1\right)}
\end{aligned}
$$

which implies $\tilde{\alpha}=\frac{2\left|x_{1}\right|-1}{\left(\left|x_{1}\right|-1\right)^{2}}=\frac{|b|-1}{c-|b|+1}$ and $\tilde{\beta}=\frac{1}{\left(\left|x_{1}\right|-1\right)^{2}}=\frac{1}{c-|b|+1}$.

(2) $c<0$. Without loss of generality, we can assume $\left|x_{1}\right| \geq\left|x_{2}\right|$, then

$$
\begin{aligned}
\tilde{\alpha} & =\sum_{i=1}^{\infty}\left|\alpha_{i}\right|=\frac{|c|}{\left|x_{1}\right|+\left|x_{2}\right|}\left(\sum_{i=1}^{\infty}\left(\frac{1}{\left|x_{1}\right|^{2 i+1}}+\frac{1}{\left|x_{2}\right|^{2 i+1}}\right)+\sum_{i=1}^{\infty}\left(\frac{1}{\left|x_{2}\right|^{2 i}}-\frac{1}{\left|x_{1}\right|^{2 i}}\right)\right) \\
& =\frac{|c|}{\left|x_{1}\right|+\left|x_{2}\right|}\left(\frac{1}{\left|x_{1}\right|\left(\left|x_{1}\right|^{2}-1\right)}+\frac{1}{\left|x_{2}\right|\left(\left|x_{2}\right|^{2}-1\right)}+\frac{1}{\left|x_{2}\right|^{2}-1}-\frac{1}{\left|x_{1}\right|^{2}-1}\right) \\
& =\frac{|c|}{\left|x_{1}\right|+\left|x_{2}\right|}\left(\frac{1}{\left|x_{2}\right|\left(\left|x_{2}\right|-1\right)}-\frac{1}{\left|x_{1}\right|\left(\left|x_{1}\right|+1\right)}\right) \\
& =\frac{\left|x_{1}\right|-\left|x_{2}\right|+1}{\left(\left|x_{1}\right|+1\right)\left(\left|x_{2}\right|-1\right)} \\
& =\frac{|b|+1}{|c|-|b|-1}
\end{aligned}
$$




$$
\begin{aligned}
\tilde{\beta} & =\sum_{i=1}^{\infty}\left|\beta_{i}\right|=\frac{1}{\left|x_{1}\right|+\left|x_{2}\right|}\left(\sum_{i=1}^{\infty}\left(\frac{1}{\left|x_{1}\right|^{2 i-1}}+\frac{1}{\left|x_{2}\right|^{2 i-1}}\right)+\sum_{i=1}^{\infty}\left(\frac{1}{\left|x_{2}\right|^{2 i}}-\frac{1}{\left|x_{1}\right|^{2 i}}\right)\right) \\
& =\frac{1}{\left|x_{1}\right|+\left|x_{2}\right|}\left(\frac{\left|x_{1}\right|}{\left|x_{1}\right|^{2}-1}+\frac{\left|x_{2}\right|}{\left|x_{2}\right|^{2}-1}+\frac{1}{\left|x_{2}\right|^{2}-1}-\frac{1}{\left|x_{1}\right|^{2}-1}\right) \\
& =\frac{1}{\left|x_{1}\right|+\left|x_{2}\right|}\left(\frac{1}{\left|x_{2}\right|-1}+\frac{1}{\left|x_{1}\right|+1}\right) \\
& =\frac{1}{|c|-|b|-1} .
\end{aligned}
$$

\section{Consecutive collinear digit set}

In the section, we characterize the connectedness of the self-affine sets $T(A, \mathcal{D})$ associated with digit sets $\mathcal{D}=\{0,1, \ldots, m\} v$. The necessary and sufficient conditions are given for $T(A, \mathcal{D})$ to be connected.

Theorem 3.1. Let the characteristic polynomial of the expanding integer matrix $A$ be $f(x)=x^{2}+b x+c$ and a digit set $\mathcal{D}=\{0,1, \ldots, m\} v$ where $m \geq 1$ is integral and $v \in \mathbb{R}^{2}$ such that $\{v, A v\}$ are linearly independent. If $\Delta=b^{2}-4 c \geq 0$ and the eigenvalues of $A$ have moduli $\geq 2$, then

(i) if $c=4$, then $T(A, \mathcal{D})$ is connected if and only if $m \geq 2$;

(ii) otherwise $c \neq 4$, then $T(A, \mathcal{D})$ is connected if and only if

$$
m \geq \begin{cases}\max \{c-|b|+1,|b|-1\} & c>0 \\ |c|-|b|-1 & c<0\end{cases}
$$

Proof. Let $T+l$ be a neighbor of $T$, then $l=\gamma v+\delta A v=\sum_{i=1}^{\infty} b_{i} A^{-i} v, b_{i} \in \Delta D=$ $\{0, \pm 1, \pm 2, \ldots, \pm m\}$. By 2.1), we have

$$
|\gamma| \leq m \tilde{\alpha} ; \quad|\delta| \leq m \tilde{\beta}
$$

Suppose $\left(T+\ell_{1} v\right) \cap\left(T+\ell_{2} v\right) \neq \emptyset$ for $0 \leq \ell_{1}<\ell_{2} \leq m$, then $\left(\ell_{2}-\ell_{1}\right) v=$ $\sum_{i=1}^{\infty} b_{i} A^{-i} v, b_{i} \in \Delta D$. By 2.2$)$, we obtain $l^{*}=-\left(\left(\ell_{2}-\ell_{1}\right) c+b_{2}\right) v-\left(b\left(\ell_{2}-\ell_{1}\right)+\right.$ $\left.b_{1}\right) A v$.

(i) If $c=4$, then $|b|=4$, by $(3.1)$ and Lemma 2.5, the connectedness of $T(A, \mathcal{D})$ can imply that 


$$
\left(\ell_{2}-\ell_{1}\right)|b|-m \leq\left|\left(\ell_{2}-\ell_{1}\right) b+b_{1}\right| \leq \frac{m}{c-|b|+1}=m
$$

which further implies that $m \geq 2\left(\ell_{2}-\ell_{1}\right) \geq 2$.

Conversely, if $b=-4$, then $f(x)=x^{2}-4 x+4$. By using $f(A)=0$ and $A f(A)=0$, we have $A^{3}-3 A^{2}+4 I=0$ and $A^{3}-A^{2}=2 A(A-I)+2(A-I)-2 I$ which yields

$$
(A-I)=2 A^{-1}(A-I)+2 A^{-2}(A-I)-2 A^{-2}
$$

and

$$
I=2 A^{-1}+2 A^{-2}-2 \sum_{i=3}^{\infty} A^{-i}
$$

Then

$$
v=2 A^{-1} v+2 A^{-2} v-2 \sum_{i=3}^{\infty} A^{-i} v \in T-T .
$$

Therefore $T$ is connected by Lemma 2.1. (see Figure 1)

If $b=4$, then $f(x)=x^{2}+4 x+4$. By Lemma 2.4, the connectedness of $T(A, \mathcal{D})$ is the same as that of $T(-A, \mathcal{D})$ in which the characteristic polynomial of $-A$ is $f(x)=x^{2}-4 x+4$.

(ii) Necessity: If $c>0$, then by (3.1) and Lemma 2.5, we have

$$
\begin{aligned}
& \left(\ell_{2}-\ell_{1}\right) c-m \leq\left|\left(\ell_{2}-\ell_{1}\right) c+b_{2}\right| \leq m \frac{|b|-1}{c-|b|+1} ; \\
& \left(\ell_{2}-\ell_{1}\right)|b|-m \leq\left|\left(\ell_{2}-\ell_{1}\right) b+b_{1}\right| \leq \frac{m}{c-|b|+1} .
\end{aligned}
$$

(3.2) implies that $m \geq\left(\ell_{2}-\ell_{1}\right)(c-|b|+1) \geq c-|b|+1$.

Now we prove $m \geq|b|-1$. Let $x_{1}, x_{2}$ denote the roots of $x^{2}+b x+c=0$, if $m<|b|-1$, then $m \leq|b|-2=\left|x_{1}\right|-1+\left|x_{2}\right|-1$. We have

$$
\frac{m}{\left(\left|x_{1}\right|-1\right)\left(\left|x_{2}\right|-1\right)} \leq \frac{1}{\left|x_{1}\right|-1}+\frac{1}{\left|x_{2}\right|-1}<2 \text {. }
$$

The last strict inequality holds due to the fact that $c>4$ and $\left|x_{1}\right|,\left|x_{2}\right| \geq 2$. From (3.3) and (3.4), it follows that

$$
2 \leq|b|-m \leq\left(l_{2}-l_{1}\right)|b|-m \leq \frac{m}{c-|b|+1}=\frac{m}{\left(\left|x_{1}\right|-1\right)\left(\left|x_{2}\right|-1\right)}<2
$$

which is a contradiction. Hence $m \geq|b|-1$.

If $c<0$, then by (3.1) and Lemma 2.5, we have

$$
\left(\ell_{2}-\ell_{1}\right)|c|-m \leq\left|\left(\ell_{2}-\ell_{1}\right) c+b_{2}\right| \leq m \frac{|b|+1}{|c|-|b|-1}
$$

implying that $m \geq\left(l_{2}-l_{1}\right)(|c|-|b|-1) \underset{8}{\geq}|c|-|b|-1$. 
Sufficiency: If $c>0$, and $m \geq \max \{c-|b|+1,|b|-1\}$, it suffices to show that $v \in T-T$ by Lemma 2.1 .

When $b<0$, by using $f(A)=A^{2}+b A+c I=0$, we have $A^{2}+b A-(b+1) I=$ $-(c+b+1) I$, i.e., $(A-I)(A+(b+1) I)=-(c+b+1) I$. It follows that

$$
I+(b+1) A^{-1}=-(c+b+1) A^{-1} \sum_{i=1}^{\infty} A^{-i} .
$$

Hence

$$
\begin{aligned}
I & =(-b-1) A^{-1}+\sum_{i=2}^{\infty}-(c+b+1) A^{-i} \\
& =(|b|-1) A^{-1}+\sum_{i=2}^{\infty}-(c-|b|+1) A^{-i} .
\end{aligned}
$$

Then $v=(|b|-1) A^{-1} v+\sum_{i=2}^{\infty}-(|c|-|b|+1) A^{-i} v \in T-T$, and $T$ is connected. When $b>0$, Lemma 2.4 and the above argument also yield that $T$ is connected. (see Figure 2)

If $c<0$, suppose $m \geq|c|-|b|-1$, then $m \geq|b|+1$ (Indeed, if $x_{1}, x_{2}$ is the roots of $x^{2}+b x+c=0$, without loss of generality, we let $\left|x_{1}\right| \geq\left|x_{2}\right|$, then $|c|-|b|-1=$ $\left.\left|x_{1} x_{2}\right|-\left(\left|x_{1}\right|-\left|x_{2}\right|\right)-1=\left(\left|x_{1}\right|+1\right)\left(\left|x_{2}\right|-1\right) \geq\left|x_{1}\right|+1>\left|x_{1}\right|-\left|x_{2}\right|+1=|b|+1\right)$.

When $b<0$, by using $f(A)=A^{2}+b A+c I=0$, we have $A^{2}+A=(-b+1)(A+$ $I)+(-c+b-1) I$. Then

$$
\begin{aligned}
I & =(-b+1) A^{-1}+(-c+b-1) \sum_{i=2}^{\infty}(-A)^{-i} \\
& =(|b|+1) A^{-1}+(|c|-|b|-1) \sum_{i=2}^{\infty}(-A)^{-i} \\
& =(|b|+1) A^{-1}+(|c|-|b|-1) \sum_{k=1}^{\infty} A^{-2 k}-(|c|-|b|-1) \sum_{k=1}^{\infty} A^{-2 k-1} .
\end{aligned}
$$

Hence $v \in T-T$ and $T$ is connected by Lemma 2.1. When $b>0$, Lemma 2.4 and the above argument also yield that $T$ is connected. (see Figure 3 )

In the proof above, the condition that eigenvalues of $A$ have moduli $\geq 2$ is essential. If otherwise, in the case that the moduli of the eigenvalues $<2$, e.g., the moduli are close to 1 , we have no idea about the conditions for $T(A, \mathcal{D})$ to be connected by estimating $\tilde{\alpha}$ or $\tilde{\beta}$.

On the other hand, if $\Delta=b^{2}-4 c<0$, it is also difficult to compute the exact values of $\tilde{\alpha}$ and $\tilde{\beta}$ in general. However, under certain special situations, the exact values of $\tilde{\alpha}$ and $\tilde{\beta}$ can still be calculated as well. 


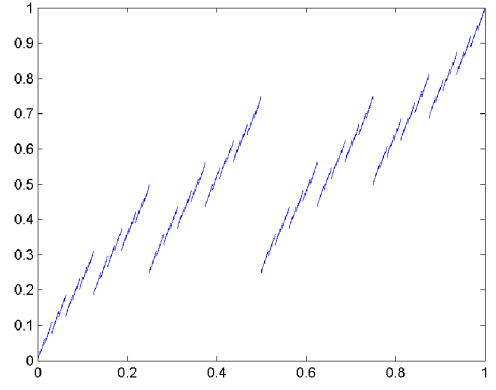

(a) $\mathrm{m}=1$

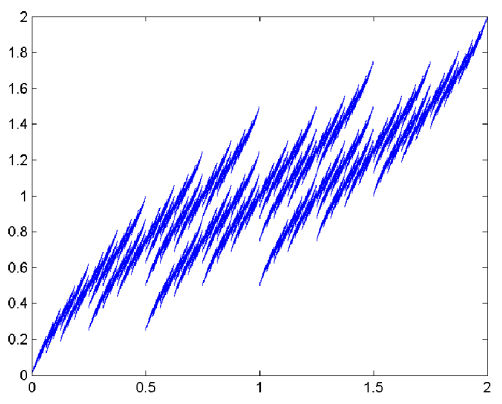

(b) $\mathrm{m}=2$

Figure 1. (a) is disconnected and (b) is connected where $A=$ $[2,0 ;-1,2], v=(1,0)^{t}$.

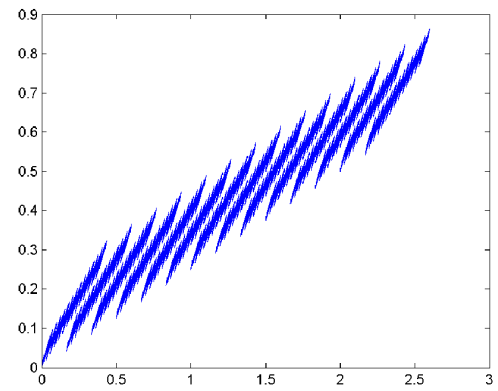

(a) $\mathrm{m}=13$

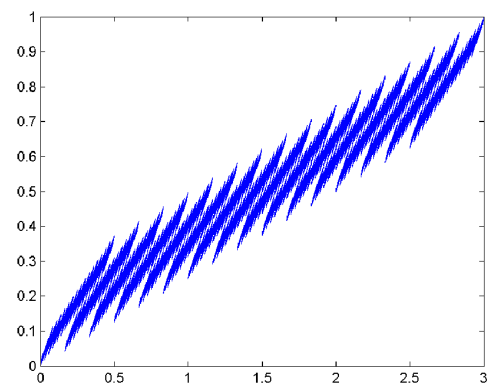

(b) $\mathrm{m}=15$

Figure 2. (a) is disconnected and (b) is connected where $A=$ $[6,0 ;-1,4], v=(1,0)^{t}$.

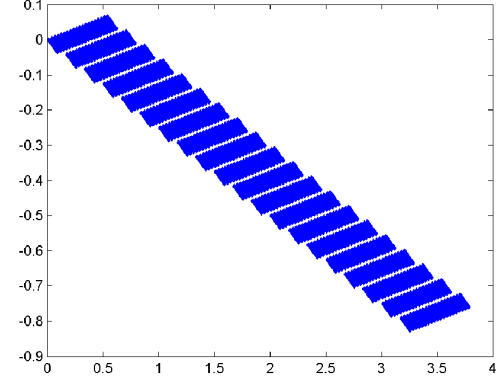

(a) $\mathrm{m}=19$

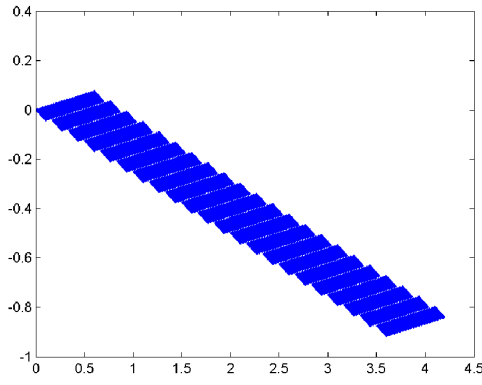

(b) $\mathrm{m}=21$

Figure 3. (a) is disconnected and (b) is connected where $A=$ $[6,0 ;-1,-4], v=(1,0)^{t}$

Theorem 3.2. Let the characteristic polynomial of $A$ be $f(x)=x^{2}+b x+c$ and $a$ digit set $\mathcal{D}=\{0,1, \ldots, m\} v$ where $m \geq 1$ and $v \in \mathbb{R}^{2}$ such that $\{v$, Av $\}$ are linearly 
independent. If $\Delta=b^{2}-4 c<0$, then $T(A, \mathcal{D})$ is connected if and only if

$$
m \geq \begin{cases}\max \{c-|b|+1,|b|-1\} & b^{2}=3 c \\ c-|b|+1 & b^{2}=2 c, b^{2}=c \\ c-1 & b=0 .\end{cases}
$$

Proof. From Lemma 2.4, we can suppose $b<0$. Let $r_{1,2}=\frac{-b \pm \sqrt{b^{2}-4 c}}{2 c}$ be the complex roots of $c x^{2}+b x+1=0$ as in Lemma 2.3 and let $r:=\left|r_{1}\right|=\left|r_{2}\right|=\frac{1}{\sqrt{c}}$. Then $r_{1}=r e^{i \theta}$ and $r_{2}=r e^{-i \theta}$ where $\theta$ is the argument of $r_{1}$. We show the necessity first by assuming $T(A, \mathcal{D})$ is connected.

(i) If $b^{2}=3 c$, then $\theta=\frac{\pi}{6}$ and

$$
\begin{gathered}
\left|\alpha_{i}\right|=\left|\frac{c\left(r_{1}^{i+1}-r_{2}^{i+1}\right)}{\Delta^{1 / 2}}\right|=\frac{2 c r^{i+1}\left|\sin \left((i+1) \frac{\pi}{6}\right)\right|}{\sqrt{c}} ; \\
\left|\beta_{i}\right|=\left|\frac{\left(r_{1}^{i+1}-r_{2}^{i+1}\right)}{\Delta^{1 / 2}}\right|=\frac{2 r^{i+1}\left|\sin \left((i+1) \frac{\pi}{6}\right)\right|}{\sqrt{c}} .
\end{gathered}
$$

Hence

$$
\begin{aligned}
\tilde{\alpha} & =\sum_{i=1}^{\infty}\left|\alpha_{i}\right|=\sqrt{c}\left(\left(\sqrt{3} r^{2}+2 r^{3}+\sqrt{3} r^{4}+r^{5}+r^{7}\right) \sum_{j=0}^{\infty} r^{6 j}\right) \\
& =\frac{3|b|^{5}+6 b^{4}+9|b|^{3}+9 b^{2}+27}{b^{6}-27}
\end{aligned}
$$

Analogous to 3.2 , we have

$$
c-m \leq\left(\ell_{2}-\ell_{1}\right) c-m \leq\left|\left(\ell_{2}-\ell_{1}\right) c+b_{2}\right| \leq m \frac{3|b|^{5}+6 b^{4}+9|b|^{3}+9 b^{2}+27}{b^{6}-27}
$$

which implies that

$$
\begin{aligned}
m & \geq \frac{b^{6}-27}{3\left(b^{4}+3|b|^{3}+6 b^{2}+9|b|+9\right)} \\
& =\frac{b^{2}}{3}-|b|+\frac{3 b^{4}+9|b|^{3}+18 b^{2}+27|b|-27}{3 b^{4}+9|b|^{3}+18 b^{2}+27|b|+27} \\
& =c-|b|+\frac{3 b^{4}+9|b|^{3}+18 b^{2}+27|b|-27}{3 b^{4}+9|b|^{3}+18 b^{2}+27|b|+27} .
\end{aligned}
$$

Thus $m \geq c-|b|+1$ as $0<\frac{3 b^{4}+9\left|b b^{3}+18 b^{2}+27\right| b \mid-27}{3 b^{4}+9|b|^{3}+18 b^{2}+27|b|+27}<1$ and $m$ is integral. (see Figure (4) 
If $|b|>3$, then $c-|b|+1>|b|-1$ is always true; if $|b|=3$ then $c=3$, and

$$
\tilde{\beta}=\sum_{i=1}^{\infty}\left|\beta_{i}\right|=\frac{1}{\sqrt{c}}\left(\left(r+\sqrt{3} r^{2}+2 r^{3}+\sqrt{3} r^{4}+r^{5}\right) \sum_{j=0}^{\infty} r^{6 j}\right)=\frac{14}{13} .
$$

Analogous to (3.3), we have

$$
3-m \leq\left(\ell_{2}-\ell_{1}\right) 3-m \leq\left|\left(\ell_{2}-\ell_{1}\right) b+b_{1}\right| \leq m \frac{14}{13}
$$

and $m \geq \frac{39}{27}$. Therefore $m \geq 2=|b|-1$.

(ii) If $b^{2}=2 c$, then $\theta=\frac{\pi}{4}$ and

$$
\left|\alpha_{i}\right|=\left|\frac{c\left(r_{1}^{i+1}-r_{2}^{i+1}\right)}{\Delta^{1 / 2}}\right|=\frac{2 c r^{i+1}\left|\sin \left((i+1) \frac{\pi}{4}\right)\right|}{|b|} .
$$

Then

$$
\tilde{\alpha}=\sum_{i=1}^{\infty}\left|\alpha_{i}\right|=|b|\left(r^{2} \sum_{j=0}^{\infty} r^{4 j}+\frac{\sqrt{2}}{2} r^{3} \sum_{k=0}^{\infty} r^{2 k}\right)=\frac{2|b|^{3}+2 b^{2}+4}{b^{4}-4} .
$$

Analogous to 3.2 , we have

$$
c-m \leq\left(\ell_{2}-\ell_{1}\right) c-m \leq\left|\left(\ell_{2}-\ell_{1}\right) c+b_{2}\right| \leq m \frac{2|b|^{3}+2 b^{2}+4}{b^{4}-4}
$$

implying that

$$
\begin{aligned}
m & \geq \frac{b^{4}-4}{2\left(b^{2}+2|b|+2\right)} \\
& =\frac{b^{2}}{2}-|b|+\frac{2 b^{2}+4|b|-4}{2 b^{2}+4|b|+4} \\
& =c-|b|+\frac{2 b^{2}+4|b|-4}{2 b^{2}+4|b|+4} .
\end{aligned}
$$

Hence $m \geq c-|b|+1$ as $0<\frac{2 b^{2}+4|b|-4}{2 b^{2}+4|b|+4}<1$ and $m$ is integral. (see Figure 5 )

(iii) If $b^{2}=c$, then $\theta=\frac{\pi}{3}$. By the similar discussion of (i) above, it follows that

$$
\tilde{\alpha}=\sum_{i=1}^{\infty}\left|\alpha_{i}\right|=\frac{b^{2}+1}{|b|^{3}-1}
$$

and

$$
m \geq \frac{|b|^{3}-1}{|b|+1}=b^{2}-|b|+\frac{|b|-1}{|b|+1}=c-|b|+\frac{|b|-1}{|b|+1} .
$$

Hence $m \geq c-|b|+1$ as $0<\frac{|b|-1}{|b|+1}<1$. (see Figure 6) 
(iv) If $b=0$, then $\theta=\frac{\pi}{2}$. Similarly, we have

$$
\tilde{\alpha}=\sum_{i=1}^{\infty}\left|\alpha_{i}\right|=\frac{c}{\sqrt{c}} \sum_{i=1}^{\infty} r^{2 i+1}=\frac{1}{c-1}
$$

and then $m \geq c-1$.

On the contrary, for the sufficiency, if $m$ satisfies (3.7), then $m \geq|b|-1$ for cases (ii) and (iii). With the similar proof as in Theorem 3.1, we can conclude that $v \in T-T$ and $T(A, \mathcal{D})$ is connected. The connectedness of case (iv) comes from the Proposition 2.2 directly.

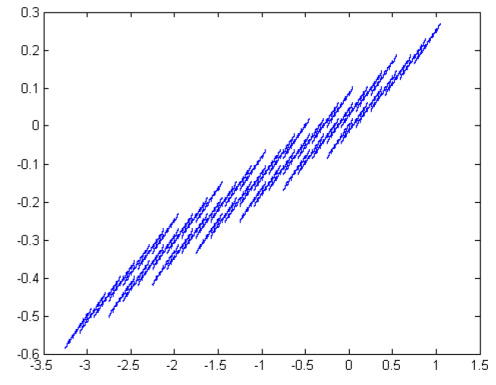

(a) $\mathrm{m}=6$

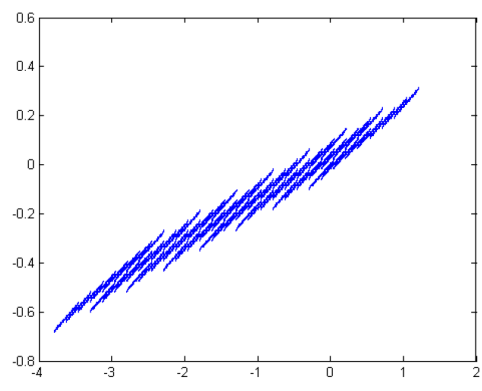

(b) $\mathrm{m}=7$

Figure 4. (a) is disconnected and (b) is connected where $A=$ $[0,-12 ; 1,-6], v=(1,0)^{t}$.

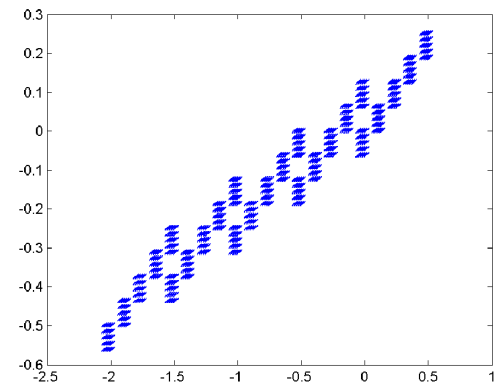

(a) $\mathrm{m}=4$

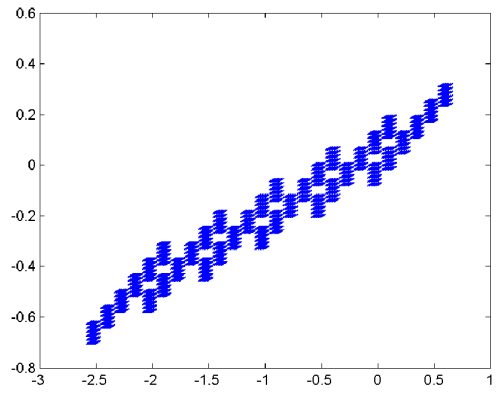

(b) $\mathrm{m}=5$

Figure 5. (a) is disconnected and (b) is connected where $A=$ $[0,-8 ; 1,-4], v=(1,0)^{t}$.

For other unsolved cases, by observing computer graphs, we conclude with the following conjecture.

Conjecture 3.3. Let the characteristic polynomial of $A$ be $f(x)=x^{2}+b x+c$ and a digit set $\mathcal{D}=\{0,1, \ldots, m\} v$ where $m \geq 1$ and $v \in \mathbb{R}^{2}$ such that $\{v, A v\}$ are linearly independent. Then 


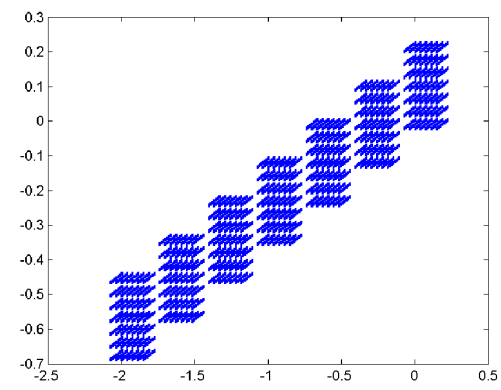

(a) $\mathrm{m}=6$

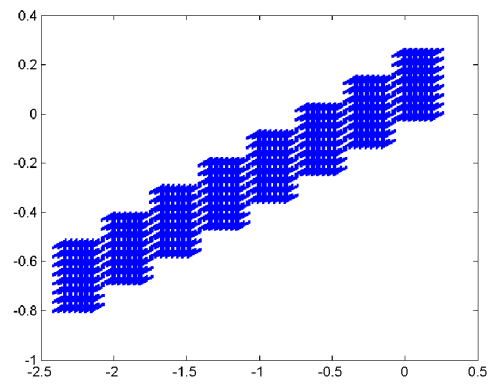

(b) $\mathrm{m}=7$

Figure 6. (a) is disconnected and (b) is connected where $A=$ $[0,-9 ; 1,-3], v=(1,0)^{t}$.

(i) if $c=|b|=4$, then $T(A, \mathcal{D})$ is connected if and only if $m \geq 2$;

(ii) otherwise, $T(A, \mathcal{D})$ is connected if and only if

$$
m \geq \begin{cases}\max \{c-|b|+1,|b|-1\} & c>0,|b| \geq 2 \\ c-1 & c>0,|b| \leq 1 \\ \max \{|c|-|b|-1,|b|+1\} & c<0 .\end{cases}
$$

\section{Non-consecutive collinear digit set}

By previous Section 2, we know that if $A \in M_{2}(\mathbb{Z})$ is an expanding matrix, then its characteristic polynomial has HRP. Let $\mathcal{D}^{\prime}=\{0,1, \ldots,(|\operatorname{det}(A)|-1)\} v$ be a consecutive collinear digit set with $\# \mathcal{D}^{\prime}=|\operatorname{det}(A)|$. By Proposition 2.2, then the associated self-affine tile $T\left(A, \mathcal{D}^{\prime}\right)$ is always connected. However there are few results on the non-consecutive collinear digit sets. In [12], Leung and one of the authors first study this case, by checking 10 eligible characteristic polynomials of the $A$ with $|\operatorname{det} A|=3$ case by case, they obtained a complete characterization for connectedness of $T(A, \mathcal{D})$ with $\mathcal{D}=\{0,1, m\} v$. In the section, we further study this kind of digit sets in more general situations. Suppose the characteristic polynomial of $A$ is of the form $f(x)=x^{2}-(p+q) x+p q$ where $|p|,|q| \geq 2$ are integers, and $\mathcal{D}=\{0,1, \ldots,|p q|-2,|p q|-1+s\} v$. By letting

$$
f_{1}(x)=x^{2} \pm 4 x+4 \quad \text { and } \quad f_{2}(x)=x^{2} \pm 7 x+12,
$$

we have the following criterion for the connectedness. 
Theorem 4.1. Let the characteristic polynomial of $A$ be $f(x)=x^{2}-(p+q) x+p q$ and a digit set $\mathcal{D}=\{0,1, \ldots,|p q|-2,|p q|-1+s\} v$ where $s \geq 0,|p|,|q| \geq 2$ are integers and $v \in \mathbb{R}^{2}$ such that $\{v, A v\}$ are linearly independent. Then

(i) if $f \neq f_{1}, f_{2}$, then $T(A, \mathcal{D})$ is connected if and only if $s=0$;

(ii) if $f=f_{1}$ or $f_{2}$, then $T(A, \mathcal{D})$ is connected if and only if $s=0$ or 1 .

Proof. If $s=0$, then $T(A, \mathcal{D})$ is always connected by Proposition 2.2 .

(i) Suppose $T(A, \mathcal{D})$ is connected, then $(T+(|p q|-1+s) v) \cap(T+i v) \neq \emptyset$ for some $0 \leq i \leq|p q|-2$. Let $r=|p q|-1+s-i$, then $r v \in T-T$, i.e., $r v=$ $\sum_{i=1}^{\infty} b_{i} A^{-i} v$ where $b_{i} \in \Delta D$. By 2.2., we obtain a new neighbor $T+l^{*}$ where $l^{*}=-\left(r p q+b_{2}\right) v+\left(r(p+q)-b_{1}\right) A v$.

If $p q>0$, then by (2.1) and Lemma 2.5, we have

$$
\begin{aligned}
&(1+s)|p q|-(|p q|-1+s) \leq r|p q|-(|p q|-1+s) \leq\left|r p q+b_{2}\right| \\
& \leq(|p q|-1+s) \frac{|p|+|q|-1}{(|p|-1)(|q|-1)} ; \\
&(1+s)|p+q|-(|p q|-1+s) \leq\left|r(p+q)-b_{1}\right| \leq \frac{|p q|-1+s}{(|p|-1)(|q|-1)} .
\end{aligned}
$$

It follows from (4.1) that

$$
s \leq \frac{|p|+|q|-2}{|p q|-|p|-|q|} .
$$

Let

$$
t=\frac{|p|+|q|-1}{(|p|-1)(|q|-1)}=\frac{1}{|p|-1}+\frac{1}{|q|-1}+\frac{1}{(|p|-1)(|q|-1)} .
$$

It is easy to see that $t<1$ if $|p|,|q| \geq 4$ or one of $|p|,|q|$ is equal to 3 and the other one is larger than 5. Therefore $|p|+|q|-2<|p q|-|p|-|q|$, and $s<1$, i.e., $s=0$. (see Figure 7)

If one of $|p|,|q|$ is equal to 2 and the other one is larger than 3, without loss of generality, suppose $|p|=2$ and $|q| \geq 3$. From (4.2) we get

$$
s \leq \frac{|q|^{2}-2|q|+2}{|q|^{2}-2}=1-\frac{2|q|-4}{|q|^{2}-2}<1 .
$$

Hence $s=0$.

If $p q<0$, analogous to the 4.1], then

$$
(1+s)|p q|-(|p q|-1+s) \leq(|p q|-1+s) \frac{|p+q|+1}{|p q|-|p+q|-1} .
$$


We have

$$
s \leq \frac{|p+q|}{|p q|-|p+q|-2}=1-\frac{|p q|-2|p+q|-2}{|p q|-|p+q|-2} .
$$

Since $p q<0$, without loss of generality, we let $|p| \geq|q|$, it follows that $|p q|-\mid p+$ $q|-1=| p q|-(|p|-|q|)-1=(|p|+1)(|q|-1) \geq| p|+1>| p|-| q|+1=| p+q \mid+1$. Thus $|p q|-2|p+q|-2>0$, and $s=0$.

(ii) If $f=f_{1}$, then (4.4) implies that $s \leq 1$ (see Figure 8); if $f=f_{2}$, then (4.3) implies that $s \leq 1$ (see Figure 9). Conversely, for $s=1$, let $\Delta D_{1}=\{0, \pm 1, \pm 2, \pm 3, \pm 4\}$ and $\Delta D_{2}=\{0, \pm 1, \ldots, \pm 12\}$ and let $A_{1}$ and $A_{2}$ denote the matrices of $f_{1}$ and $f_{2}$ respectively. We only need to show that $v, 2 v \in T-T$ (see [9] or [12]). Let $\Delta D_{1}^{\prime}=\{0, \pm 1, \pm 2\}$ and $\Delta D_{2}^{\prime}=\{0, \pm 1, \ldots, \pm 6\}$. By Theorem 3.1, there exist sequences $\left\{b_{1 i}\right\}_{i=1}^{\infty}$ where $b_{1 i} \in \Delta D_{1}^{\prime}$ and $\left\{b_{2 i}\right\}_{i=1}^{\infty}$ where $b_{2 i} \in \Delta D_{2}^{\prime}$ such that $v=$ $\sum_{i=1}^{\infty} b_{1 i} A_{1}^{-i} v \in T-T$ and $v=\sum_{i=1}^{\infty} b_{2 i} A_{2}^{-i} v \in T-T$. Moreover, $2 b_{1 i} \in \Delta D_{1}$ and $2 b_{2 i} \in \Delta D_{2}$. Hence $2 v \in T-T$ as well.

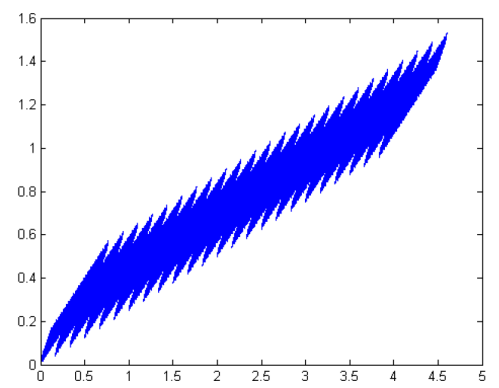

(a) $\mathrm{s}=0$

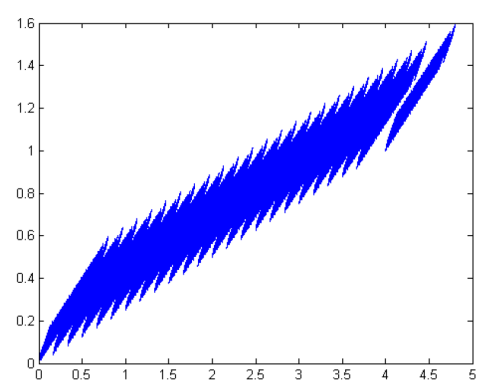

(b) $\mathrm{s}=1$

Figure 7. (a) is connected and (b) is disconnected where $A=$ $[6,0 ;-1,4], v=(1,0)^{t}$.

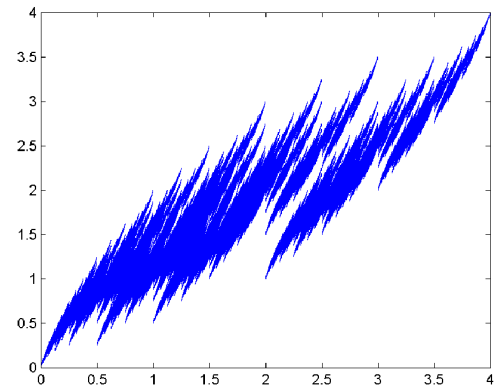

(a) $\mathrm{s}=1$

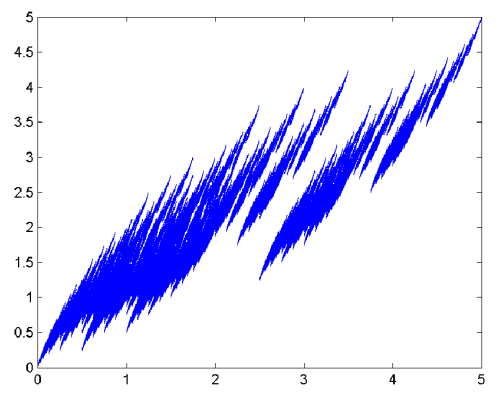

(b) $s=2$

Figure 8. (a) is connected and (b) is disconnected where $A=$ $[2,0 ;-1,2], v=(1,0)^{t}$. 


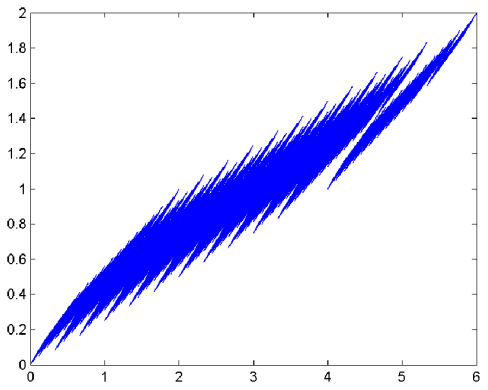

(a) $\mathrm{s}=1$

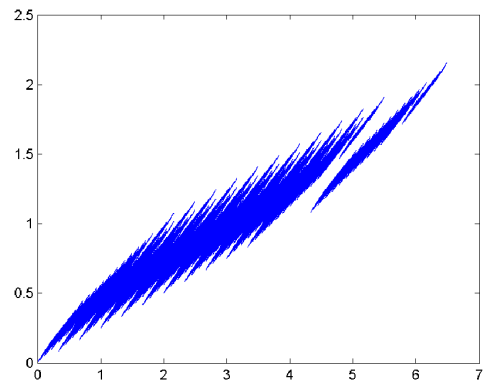

(b) $\mathrm{s}=2$

Figure 9. (a) is connected and (b) is disconnected where $A=$ $[3,0 ;-1,4], v=(1,0)^{t}$.

\section{References}

[1] S. Akiyama And N. Guini, On the connectedness of self-affine tiles, Arch. Math. 82(2004), 153-163.

[2] C. Bandt and Y. Wang, Disk-like self-affine tiles in $\mathbb{R}^{2}$, Discrete Comput. Geom. 26(2001), 591-601.

[3] Q.R. Deng And K.S. Lau, Connectedness of a class of planar self-affine tiles, J. Math. Anal. Appl. 380(2011), 493-500.

[4] K. Gröchenig And A. HaAs, Self-similar lattice tilings, J. Fourier Anal. Appl. 1(1994), 131-170.

[5] D. Hacon, N.C. Saldanha and J.J.P. Veerman, Remarks on self-affine tilings, Experiment. Math. 3(1994), 317-327.

[6] M. Hata, On the structure of self-similar sets, Japan J. Appl. Math. 2(1985), 381-414.

[7] X.G. He, I. Kirat AND K.S. LAU, Height reducing property of polynomials and self-affine tiles, Geom. Dedicata 152(2011), 153-164.

[8] I. KIRAT, Disk-like tiles and self-affine curves with non-collinear digits, Math. Comp. 79(2010), 1019-1045.

[9] I. Kirat and K.S. Lau, On the connectedness of self-affine tiles, J. London Math. Soc. (2) 62(2000), 291-304.

[10] I. KIRAT, K.S. LAU AND H. RAO, Expanding polynomials and connectedness of self-affine tiles, Discrete Comput. Geom. 31(2004), 275-286.

[11] K.S. Leung and K.S. Lau, Disk-likeness of planar self-affine tiles, Trans. Amer. Math. Soc. 359(2007), 3337-3355.

[12] K.S. Leung AND J.J. Luo, Connectedness of planar self-affine sets associated with nonconsecutive collinear digit sets, J. Math. Anal. Appl. 395(2012), 208-217.

[13] K.S. Leung AND J.J. Luo, Connectedness of planar self-affine sets associated with noncollinear digit sets, (2014) submitted.

[14] K.S. Leung And J.J. Luo, Boundaries of disk-like self-affine tiles, Discrete Comput Geom (2013) 50:194C218.

[15] J. C. Lagarias And Y. Wang, Self-affine tiles in $\mathbb{R}^{n}$, Adv. Math. 121(1996), 21-49. 
1 Key Laboratory of High Performance Computing and Stochastic Information Processing (Ministry of Education of China), College of Mathematics and Computer Science, Hunan Normal University, Changsha, Hunan 410081, China

E-mail address: liujingcheng11@126.com

2 Department of Mathematics, Shantou University, Shantou 515063, Guangdong, P.R. CHINA

E-mail address: jasonluojun@gmail.com

E-mail address: luojun@stu.edu.cn 